\title{
Qingjie Fuzheng Granules regulates cancer cell proliferation, apoptosis and tumor angiogenesis in colorectal cancer xenograft mice via Sonic Hedgehog pathway
}

\author{
Xiao-Qin Zhu ${ }^{1,2}$, Hong Yang ${ }^{1,2}$, Ming-He Lin ${ }^{3}$, Hai-Xia Shang ${ }^{1,2}$, Jun Peng ${ }^{1,2}$, Wu-Jin Chen ${ }^{4}$, \\ Xu-Zheng Chen ${ }^{1,2}$, Jiu-Mao Lin ${ }^{1,2}$ \\ ${ }^{1}$ Academy of Integrative Medicine, Fujian University of Traditional Chinese Medicine, Fuzhou, China; ${ }^{2}$ Fujian Key Laboratory of Integrative \\ Medicine on Geriatrics, Fuzhou, China; ${ }^{3}$ Editorial Department of Rehabilitation Medicine, Fujian University of Traditional Chinese Medicine, \\ Fuzhou, China; ${ }^{4}$ Department of Oncology, Affiliated People's Hospital of Fujian University of Traditional Chinese Medicine, Fuzhou, China \\ Contributions: (I) Conception and design: XQ Zhu, H Yang; (II) Administrative support: MH Lin, J Peng; (III) Provision of study materials: HX \\ Shang, WJ Chen; (IV) Collection and assembly of data: XZ Chen; (V) Data analysis and interpretation: JM Lin; (VI) Manuscript writing: All authors; \\ (VII) Final approval of manuscript: All authors. \\ Correspondence to: Jiu-Mao Lin, PhD. Academy of Integrative Medicine, Fujian University of Traditional Chinese Medicine, 1 Qiuyang Road, Minhou \\ Shangjie, Fuzhou 350122, China. Email: linjiumao@fjtcm.edu.cn.
}

Background: Sonic Hedgehog (SHh) signaling pathway plays a critical role in cell proliferation, apoptosis, and tumor angiogenesis in various types of malignancies including colorectal cancer (CRC). Qingjie Fuzheng Granules (QFG) is a traditional Chinese medicinal formula, which has been clinically used in various cancer treatments, including CRC. In this study, we explored the potential molecular mechanisms of QFG treatment effects on $\mathrm{CRC}$ via the $\mathrm{SHh}$ pathway.

Methods: A CRC HCT-116 xenograft mouse model was utilized for all experiments. Mice were treated with intra-gastric administration of $1 \mathrm{~g} / \mathrm{kg}$ of QFG or saline 6 days a week for 28 days (4 weeks). Body weight, length and shortest diameter of the tumor were measured every 3 days. At the end of the treatment, the tumor weight was measured. TUNEL staining assays were used to detect tumor apoptosis. Western blot and immunohistochemistry (IHC) assays were used to detect the expression of relative proteins.

Results: In our results, QFG inhibited the increase of tumor volume and weight, and exhibited no impact on mouse body weight. Furthermore, QFG significantly decreased the expression of SHh, Smo and Gli proteins, indicating the action of SHh signaling. Consequently, the expression of pro-proliferative survivin, Ki-67, Cyclin-D1 and CDK4 were decreased and expression of anti-proliferative p21 was increased. The pro-apoptotic Bax/Bcl-2 ratio, cle-caspase-3 and TUNEL-positive cell percentage in tumor tissues were increased. Meanwhile, the pro-angiogenic VEGF-A and VEGFR-2 expression was down-regulated.

Conclusions: QFG inhibited CRC cell proliferation and promoted CRC cell apoptosis and tumor angiogenesis in vivo through the suppression of SHh pathway, suggesting that QFG could be a potential therapeutic drug for CRC.

Keywords: Qingjie Fuzheng Granules (QFG); colorectal cancer (CRC); proliferation; apoptosis; angiogenesis

Submitted May 19, 2020. Accepted for publication Sep 25, 2020.

doi: 10.21037/jgo-20-213

View this article at: http://dx.doi.org/10.21037/jgo-20-213 


\section{Introduction}

Owing to diet structure and lifestyle changes, as well as the growing aging population, colorectal cancer (CRC) has become the third most common malignancies in the world $(1,2)$. Surgery and chemoradiotherapy, the primary clinical treatment methods for CRC, can effectively control the development of CRC. However, long-term surgery and chemoradiotherapy may cause recurrence, metastasis and significantly reduce the quality of life for patients (3). Thus, the search for new therapies with less toxic side effects has garnered considerable interest worldwide.

Traditional Chinese medicines have some advantages in this regard. Qingjie Fuzheng Granules (QFG) is a traditional Chinese medicine (TCM) formula used extensively as an alternative medicine for cancer treatment. QFG administration is clinically effective for CRC treatment and has been shown to significantly improve the immune function of CRC patients with few adverse effects (4). It is formulated of four herbs, including Hedyotis diffusa Willd, Malt, Astragalus, and Scutellaria barbata D.Don. In a previous study, we demonstrated that QFG inhibits proliferation and promotes apoptosis in liver cancer cells in vitro (5). Additionally, we found that QFG also obtained the inhibitory effect on CRC cells in vitro (6). Moreover, QFG was shown to decrease the cancer treatment drug fluorouracil (5-FU)-induced severe intestinal mucosal damages and diarrhea, by improving intestinal barrier function, inhibiting cell apoptosis and the expression of pro-inflammatory cytokines, promoting cell proliferation and the expression of anti-inflammatory cytokines, without affecting anti-tumor effect of 5-FU (7). However, the potential molecular mechanisms of QFG warrant further investigation through in vivo research.

Notably, over-proliferation and inadequate apoptosis in tumors is the main contributor to cancer development. Dysregulated cell cycle would cause uncontrolled cell proliferation, and cancer is a disease of inappropriate cell proliferation (8). Apoptosis ensures proper body maintenance and function by removing redundant and abnormal cells $(9,10)$. Apoptosis dysfunction acts as one of the triggers of several diseases, including CRC (11). Furthermore, angiogenesis plays a vital role in the growth and development of cancer (12). Angiogenesis is the anatomical basis and precondition for tumor blood supply and a necessary condition for growth, metastasis, and diffusion of tumors (12). Thus, some of the crucial ways of exploring antitumor drugs is targeting suppression of proliferation, angiogenesis, and acceleration of apoptosis that lead to cancer growth.
The Sonic Hedgehog ( $\mathrm{SHh}$ ) signaling pathway is one of many that regulate the process of cancer growth and metastasis. The transduction process of the SHh signaling pathway is related to Hedgehog (Hh) ligands ( $\mathrm{SHh}, \mathrm{DHh}$ and $\mathrm{IHh})$, membrane proteins (Smo) and transcription factors (Gli1, Gli2 and Gli3) (13). Smo is a transmembrane protein, usually in the form of a complex, at this time the activity of Smo is suppressed (13). When SHh signaling is activated in the cell, the Smo complex is degraded and Smo is reactivated. The activated Smo promotes Gli1 activator travelling to the nucleus and activates the transcription of downstream target genes, such as Cyclin D, Bcl-2, Bax, VEGF, etc., thereby regulating cell cycleinduced cell proliferation, reducing apoptosis, promoting angiogenesis and eventually leading to tumorigenesis and tumor development (14-16). Thus, inhibition of proliferation, angiogenesis and acceleration of apoptosis via modulation of SHh signaling could be a major focus for anticancer drug development. The aim of the present study is to demonstrate the anti-cancer activities of QFG by investigating the underlying molecular mechanisms in a CRC mouse xenograft model. We present the following study in accordance with the ARRIVE reporting checklist (available at http://dx.doi.org/10.21037/jgo-20-213).

\section{Methods}

\section{Preparation of $Q F G$}

QFG were provided by the Academy of Pharmacy of Fujian University of Traditional Chinese Medicine (Fuzhou, Fujian, China) and stored at $4^{\circ} \mathrm{C}$. For all experiments, $\mathrm{QFG}$ powder was dissolved in saline (R22173, Yuanye Biology, Shanghai, China).

\section{Cell culture}

We purchased human colon carcinoma HCT-116 cell line from the cell resource center of Shanghai institute of life sciences, Chinese academy of sciences. HCT-116 cells were grown in RPMI-1640 complete medium (C11875500BT, Life Technologies Corp., Grand Island, NY, USA), containing $10 \% \mathrm{FBS}, 1 \%$ penicillin/streptomycin, and a humidified atmosphere with the condition of $5 \% \mathrm{CO}_{2}$ at $37^{\circ} \mathrm{C}$.

\section{Animal models and treatment}

In this study, $20 \mathrm{SPF}$ grade, six-week-old male BALB/c nude mice (weight: 20-22 g) were purchased from SLAC Laboratory Animal Technology Co., Ltd. (Shanghai, 
China). All mice were housed in specific pathogenfree conditions with controlled temperature $\left(23 \pm 2{ }^{\circ} \mathrm{C}\right)$ and humidity $(60 \% \pm 10 \%)$ under a 12 -h light/dark cycle with ad libitum access to food and water. The mice were fed adaptively for 3-4 days and the following xenograft implantation experiments were carried out. After mixing the HCT-116 cells $\left(2 \times 10^{6}\right)$ with an equal volume of PBS and Matrigel (354248, Corning, Shanghai, China), $0.1 \mathrm{~mL}$ mixture was injected into the right flank area of each mouse subcutaneously. When the size of the tumor was approximately at $100-300 \mathrm{~mm}^{2}$, mice were randomly categorized into control group and QFG-treated group ( $\mathrm{n}=10$ for each group). While the control group was given intragastric administration of saline, the QFG-treated group was given $1 \mathrm{~g} / \mathrm{kg}$ of QFG, 6 days a week for 28 days (4 weeks). We measured the body weight, the length (A) and shortest (B) diameter of the tumor from 20 mice every 3 days with an electronic balance and a caliper. We calculated the tumor volume using the following formula: $\mathrm{V}=\left(\mathrm{A} \times \mathrm{B}^{2}\right) / 2$. After the 28-day treatment, all mice were sacrificed by cervical dislocation under anesthesia with ether. Six tumor tissues per group were selected randomly, weighed and fixed with $4 \%$ paraformaldehyde for terminal deoxynucleotidyl transferase-mediated dUTP nick end labeling (TUNEL) and Immunochemistry (IHC) assay. Four tumor tissues per group were selected randomly for following Western blot assay. The animal experimental procedures of this study were performed strictly according to international ethical guide lines and complied with the Guidance Suggestions for the Care and Use of Laboratory Animals issued by the Ministry of Science and Technology of the People's Republic of China. It was approved by Animal Care and Use Committee of Fujian University of Traditional Chinese Medicine (No. 2019009).

\section{TUNEL staining assay}

We conducted TUNEL staining assays with an in-situ apoptosis detection kit (TAK-MK500-KI01, Enzo Life Sciences, Farmingdale, USA) per the manufacturer's instructions. We considered brown stained cells as apoptotic cells. Three samples were selected from each group. The quantification of this assay was evaluated through the ratio of apoptotic cell number to a total cell number in five fields, which were randomly selected in each slides and were observed blindly by three independent observers.

\section{Immunobistochemistry assay}

We performed immunohistochemistry (IHC) assays as reported previously (7). Briefly, $5 \mu \mathrm{m}$ slices from Paraffinembedded tumor tissues were treated with primary antibodies [Ki-67 (\#9027), cle-caspase-3 (\#9662), Bcl2 (\#4223), Bax (\#5023), Cell Signaling Technology, 1:500, Beverly, MA, USA); survivin (10508-1-AP), p2 1 (10355-1-AP), CD31 (11265-1-AP), VEGF-A (190031-AP), VEGFR-2 (26415-1-AP), Protein Tech Group, 1:200, Wuhan, China); Cyclin-D1 (sc-753), CDK4 (sc16637), SHh (sc-365122), and Gli (sc-515781), Santa Cruz Biotechnology, 1:500, Santa Cruz, CA, USA] overnight at $4{ }^{\circ} \mathrm{C}$. Then, all sections were incubated with a corresponding secondary antibody for $30 \mathrm{~min}$ at room temperature and treated with the $A B C$ reagent for $30 \mathrm{~min}$. Next, sections were treated with 3,3'-diaminobenzidine for $10 \mathrm{~min}$. Finally, the detected indexes were observed and documented $(\times 400)$ by a microscope (Leica, Solms, Germany). Three samples were selected from each group. The quantification of IHC assays was evaluated through the ratio of positive cell number to a total cell number in five fields, which were randomly selected in each slides and were observed blindly by three independent observers.

\section{Western blot assay}

Four tumor tissues were selected randomly from control or QFG group, homogenized in lysis buffer. Total protein concentrations were estimated by a BCA assay kit (Pierce, MA, USA). Total protein $(30 \mu \mathrm{g})$ was used for electroblotting and transferred to NC membranes (Millipore, MA, USA). The membranes were blocked by $5 \%$ skim milk, and incubated with primary antibodies [SHh (bs-1544R), Smo (bs-2801R), Gli (bs-1206R), Bioss, 1:500, Wuhan, China] and $\beta$-actin (\#4967, Cell Signaling Technology, 1:1,000, Beverly, MA, USA) at $4{ }^{\circ} \mathrm{C}$ overnight. Then, appropriate HRP-conjugated secondary antibodies: goat anti-mouse IgG secondary antibody (\#L3032, Signalway Antibody, 1:5,000, PA, USA) and goat anti-rabbit IgG secondary (\#L3012, Signalway Antibody, 1:5,000, PA, USA) were incubated with these membranes for 1 hour at room temperature. Finally, SuperSignal West Pico Chemiluminescent Substrate was used to detect the immunoreactive protein signals. Protein expression was estimated by measuring intensity of exposure with densitometry in an averaged over three experiments. 


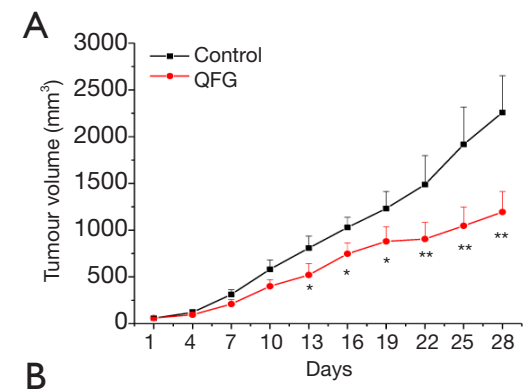

B
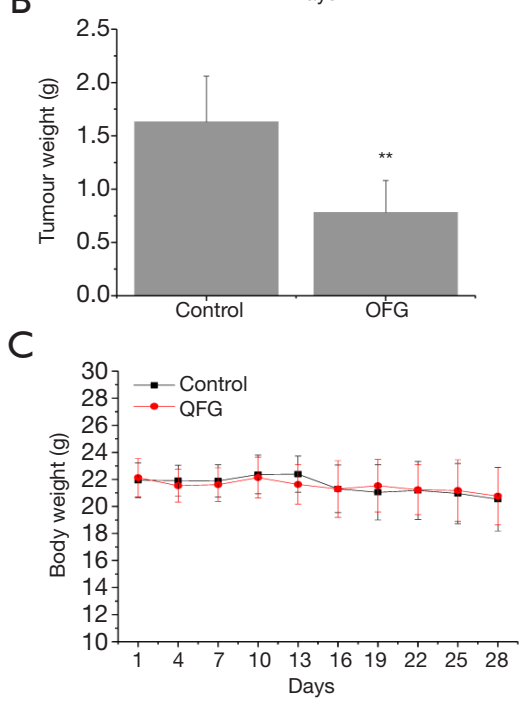

Figure 1 Effect of QFG on tumor growth in CRC xenograft mice. (A) Tumor volume $\left(\mathrm{mm}^{3}\right)$ of a nude mouse was measured every 3 days for 28 days (4 weeks). (B) The tumor weight (g) of a nude mouse was measured at the end of the study. (C) The body weight ( $\mathrm{g}$ ) of a nude mouse was measured every 3 days for 28 days (4 weeks). Data are expressed as averages with S.D. (error bars), $\mathrm{n}=10$ each group. ${ }^{*} \mathrm{P}<0.05,{ }^{*} \mathrm{P}<0.01, \mathrm{QFG}$ treatment group $v$. control group. CRC, colorectal cancer; QFG, Qingjie Fuzheng Granules.

\section{Statistical analysis}

The data analysis was performed with SPSS software (version 21.0). Measurement data is expressed as mean \pm standard deviation. The relationship between two groups was analyzed using unpaired $t$-test and the statistical analysis in two or more variables was tested by one-way ANOVA. Statistically significant result was labeled by $\mathrm{P}<0.05$.

\section{Results}

QFG inhibits tumor growth in CRC xenograft mice

To assess the effect of QFG on tumor growth, we determined the tumor volume and weight as well as body weight in all CRC xenograft mice $(n=20)$, and then compared these data in the QFG-treated group with the control group. The tumor volume and weight in the QFG-treated group on day 28 were $1,193.74 \pm 219.56 \mathrm{~mm}^{3}$ and $0.782 \pm 0.3 \mathrm{~g}$, respectively, whereas those in the control group were $2,258.57 \pm 393.57 \mathrm{~mm}^{3}$ and $1.63 \pm 0.43 \mathrm{~g}$, respectively $(\mathrm{P}<0.01$; Figure $1 A, B)$. The body weight exhibited no significant difference in the QFG-treated group compared with the control group (Figure 1C). Overall, these findings suggested that QFG was effective in inhibiting CRC tumor growth, without significant effect on body weight of CRC xenograft mice.

\section{QFG inbibits cancer cell proliferation in CRC xenograft mice}

The impact of QFG on CRC tumor cell proliferation was determined using IHC assays for $\mathrm{Ki}-67$, a proliferation marker specifically expressed in proliferating cell nuclei (17). In the control group, the percentage of Ki-67positive cells in CRC tumors was $40.28 \% \pm 14.17 \%$, whereas those in the QFG-treated group was $18.63 \% \pm 8.90 \%$ $(\mathrm{P}<0.01)$, suggesting that $\mathrm{QFG}$ inhibited proliferation of CRC tumor cells (Figure 2).

During the cell cycle process, survivin is a vital marker which can indicate the degree of cell-cycle progression, and Cyclin-D1 binds to its specific ligand CDK4 to form a complex to elicit pro-proliferative function early through the G1/S phase $(18,19)$. On the contrary, p21 is a negative regulator of cell-cycle (20). In this study, we assessed the effect of QFG on these cell-cycle-regulatory factors. QFG treatment decreased the expression level of survivin, Cyclin-D1, and CDK4 in tumor tissues, whereas treatment increased the expression of p21 (Figure 2). These findings further validated that the effect of QFG could inhibit CRC tumor cell proliferation.

\section{QFG induces cancer cell apoptosis in CRC xenograft mice}

We examined apoptosis in tumors using the TUNEL assay. The percentage of TUNEL-positive cells in tumor tissues in the control group was $6.9 \% \pm 2.37 \%$, whereas that in the QFG-treated group was $30.0 \% \pm 3.49 \%(\mathrm{P}<0.01$; Figure 3$)$. In addition, we observed that $\mathrm{QFG}$ up-regulated the expression of cle-caspase- 3 in CRC tumor cells $(\mathrm{P}<0.01$; Figure 3), demonstrating that $\mathrm{QFG}$ induced apoptosis in vivo. We determined the effects of $\mathrm{QFG}$ on the expression of Bax and Bcl-2, key regulators of apoptosis, to further 

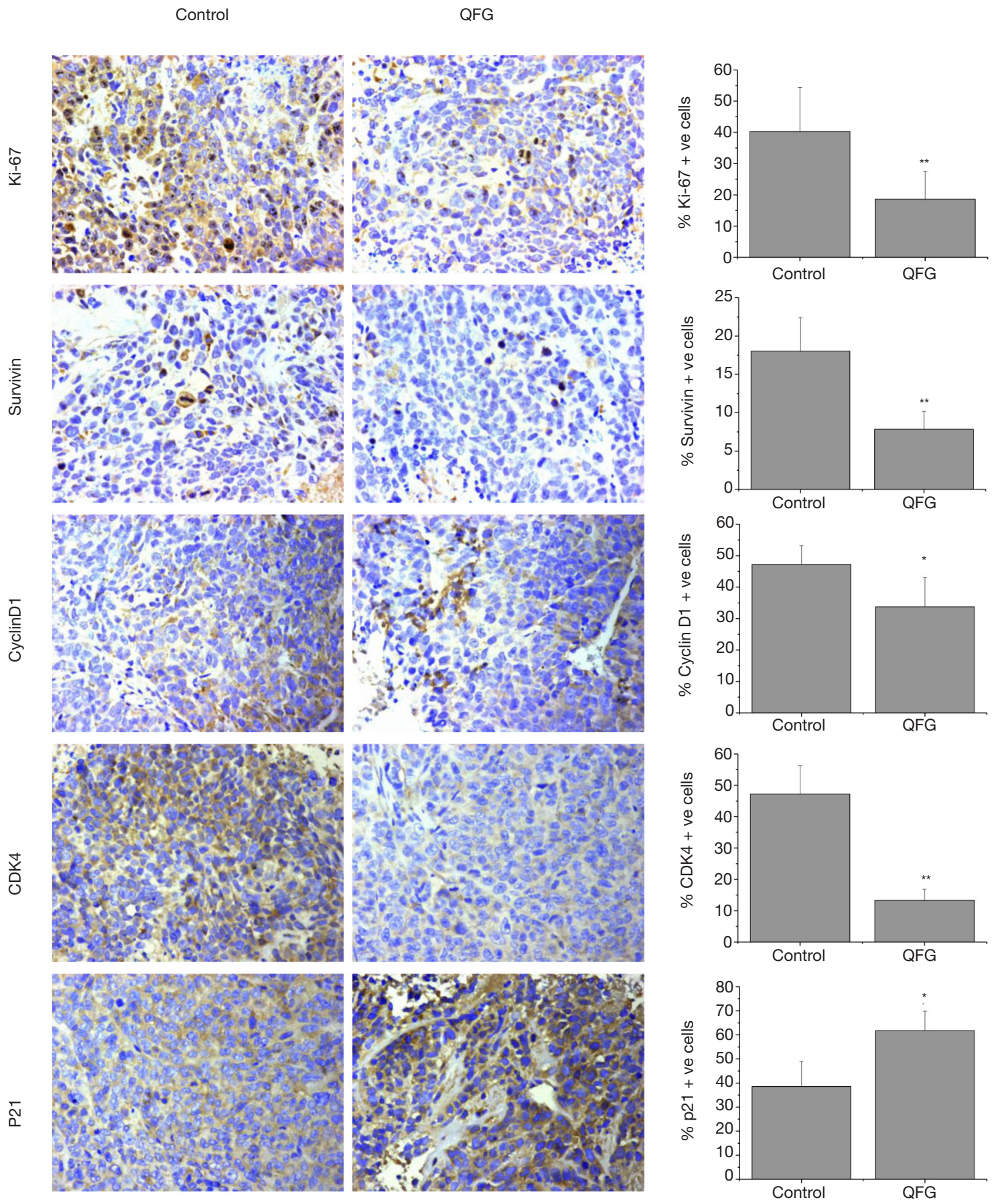

Figure 2 Effect of QFG on tumor cell proliferation in CRC xenograft mice. Expression of Ki-67, survivin, Cyclin-D1, CDK4, and p21 of tumor tissues were detected by IHC assays $(\times 400)$. The percentage of positively stained cells are used as the basis for quantification in IHC assay. Data are expressed as averages with S.D. (error bars), $\mathrm{n}=3$ each group. ${ }^{*} \mathrm{P}<0.05,{ }^{* *} \mathrm{P}<0.01, \mathrm{QFG}$ treatment group vs. control group. CRC, colorectal cancer; QFG, Qingjie Fuzheng Granules. 

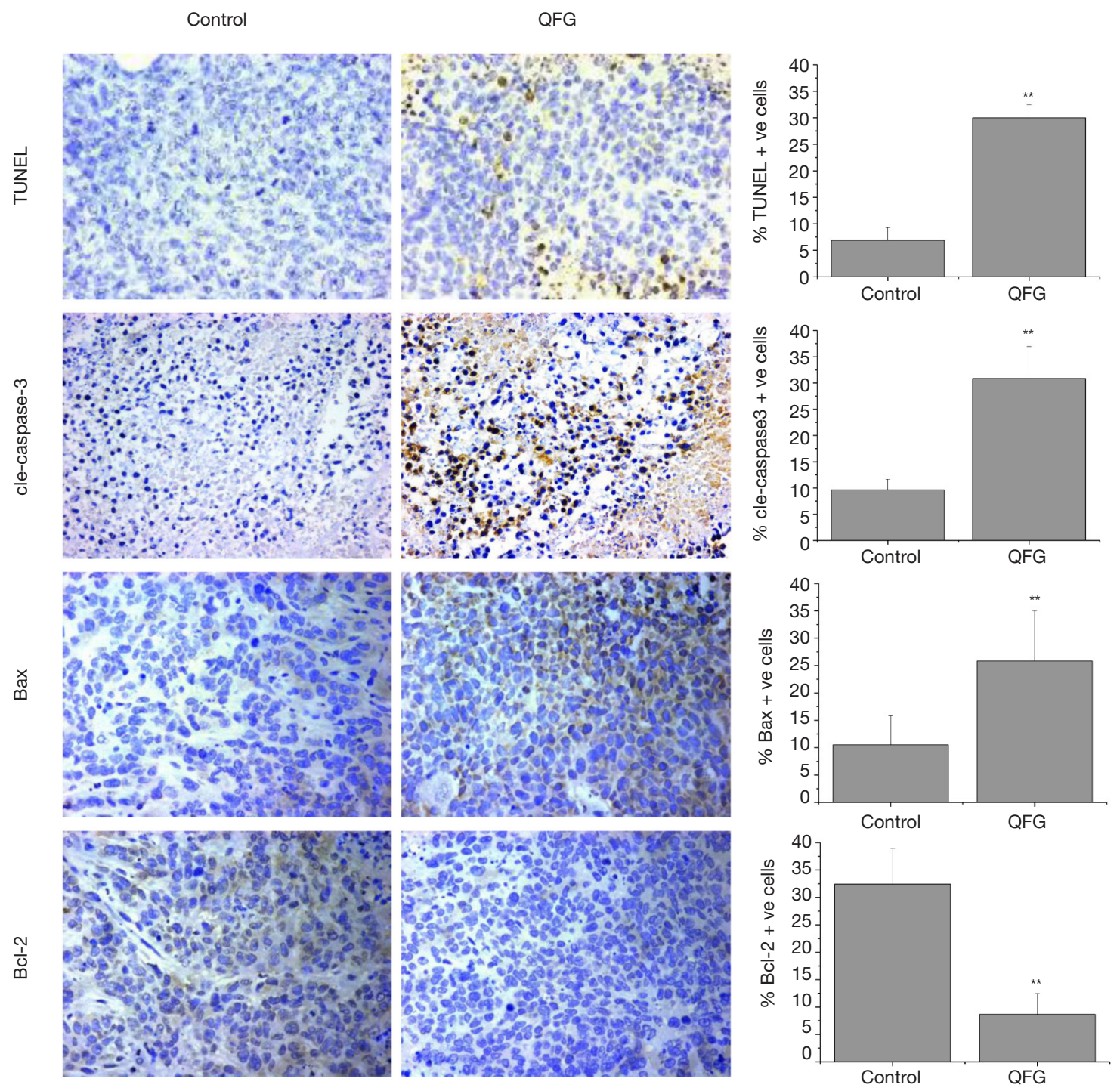

Figure 3 Effect of QFG on tumor cell apoptosis in CRC xenograft mice. Tumor cells apoptosis was detected by TUNEL staining assays (×400). The expressions of cle-caspase-3, Bax, and Bcl-2 of tumor tissues were detected by IHC assays ( $\times 400)$. The percentage of positively stained cells are used as the basis for quantification in TUNEL staining and IHC assays. Data are expressed as averages with S.D. (error bars), $\mathrm{n}=3$ each group. ${ }^{*} \mathrm{P}<0.01$, Significance is calculated as $\mathrm{QFG}$ treatment group vs. control group. CRC, colorectal cancer; QFG, Qingjie Fuzheng Granules.

verify this result. Compared with the control group, QFG up-regulated the Bax expression while down-regulating the Bcl-2 expression $(\mathrm{P}<0.01$ in both cases; Figure 3). These results showed that QFG could induce CRC tumor cell apoptosis.

\section{QFG inhibits tumor angiogenesis in CRC xenograft mice}

During angiogenesis regulation, CD31 is a key marker that indicates angiogenesis progression, because it can reflect the micro vessel density (21). Thus, we used IHC to detect CD31 expression (Figure 4). The percentage of CD31-positive cells in CRC tumors was $29.75 \% \pm 5.26 \%$, whereas that in the QFG-treated group was $12.25 \% \pm 2.92 \%$ $(\mathrm{P}<0.01)$, indicating that $\mathrm{QFG}$ inhibited CRC tumor cell angiogenesis.

In addition, VEGF-A and VEGFR-2 are critical regulated factors for angiogenesis (22). We used IHC to detect the 

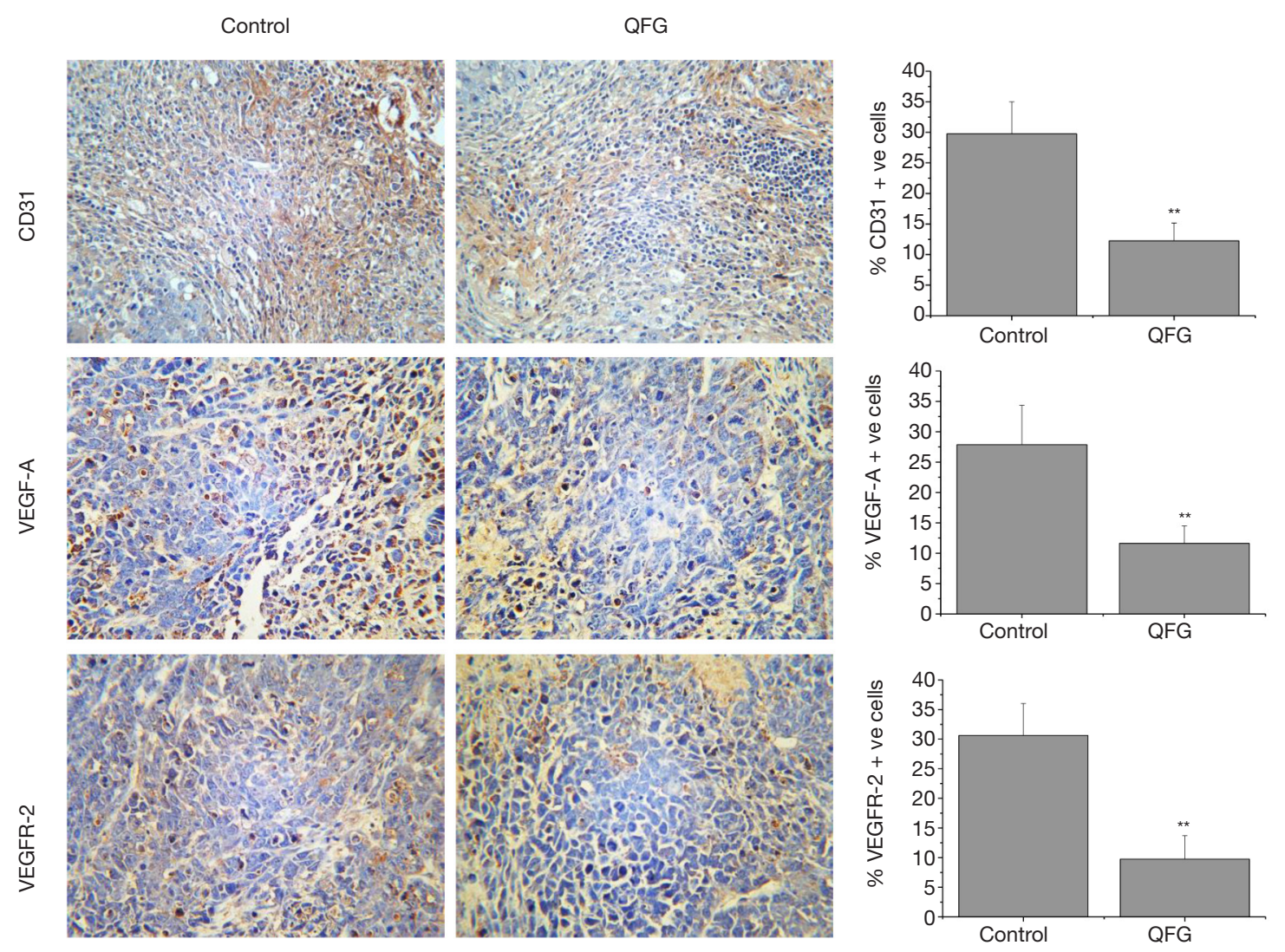

Figure 4 Effect of QFG on tumor angiogenesis in CRC xenograft mice. The expression of CD31, VEGF-A, and VEGFR-2 of tumor tissues were detected by IHC assays $(\times 400)$. The percentage of positively stained cells are used as the basis for quantification in IHC assay. Data are expressed as averages with S.D. (error bars), $\mathrm{n}=3$ each group. ${ }^{* *} \mathrm{P}<0.01$, QFG treatment group vs. control. CRC, colorectal cancer; QFG, Qingjie Fuzheng Granules.

expression of VEGF-A and VEGFR-2 to further investigate the inhibition effects of QFG on CRC tumor angiogenesis. The QFG treatment down-regulated the proteins VEGF-A and VEGFR-2 in CRC tumors $(\mathrm{P}<0.01)$, thus proving that $\mathrm{QFG}$ can inhibit angiogenesis in CRC tumors (Figure 4).

\section{QFG suppresses SHb signaling pathway in CRC xenograft mice}

Finally, we detected the expression of some SHh signaling vital regulators in CRC xenograft tissues to further investigate the potential mechanism of the inhibition effects of QFG on CRC growth. In the control group, the respective percentages of SHh-positive, and Glipositive cells in saline treated CRC xenograft tissues were $20.92 \% \pm 3.00 \%$ and $19.86 \% \pm 3.06 \%$, whereas positive cells in the QFG-treated group were $8.70 \% \pm 2.00 \%$ and
$5.40 \% \pm 2.52 \%(\mathrm{P}<0.01$; Figure $5 A)$. We confirmed these results by detecting expression level changes of relevant protein after QFG treatment: the expression levels of SHh, Smo and Gli were significantly decreased by QFG treatment $(\mathrm{P}<0.05$; Figure $5 B, C)$. These findings indicate that $\mathrm{QFG}$ treatment suppresses $\mathrm{SHh}$ signaling pathway to inhibit the growth of CRC.

\section{Discussion}

CRC is a common digestive system malignant tumor, which seriously affects human health and survival. Due to the aging population, unfavorable modern dietary habits, and increased risk factors such as smoking, lack of exercise, and obesity, the incidence and mortality of CRC are increasing year by year (23). At present, CRC is treated primarily by surgery, radiotherapy and chemotherapy (24). However, 
A
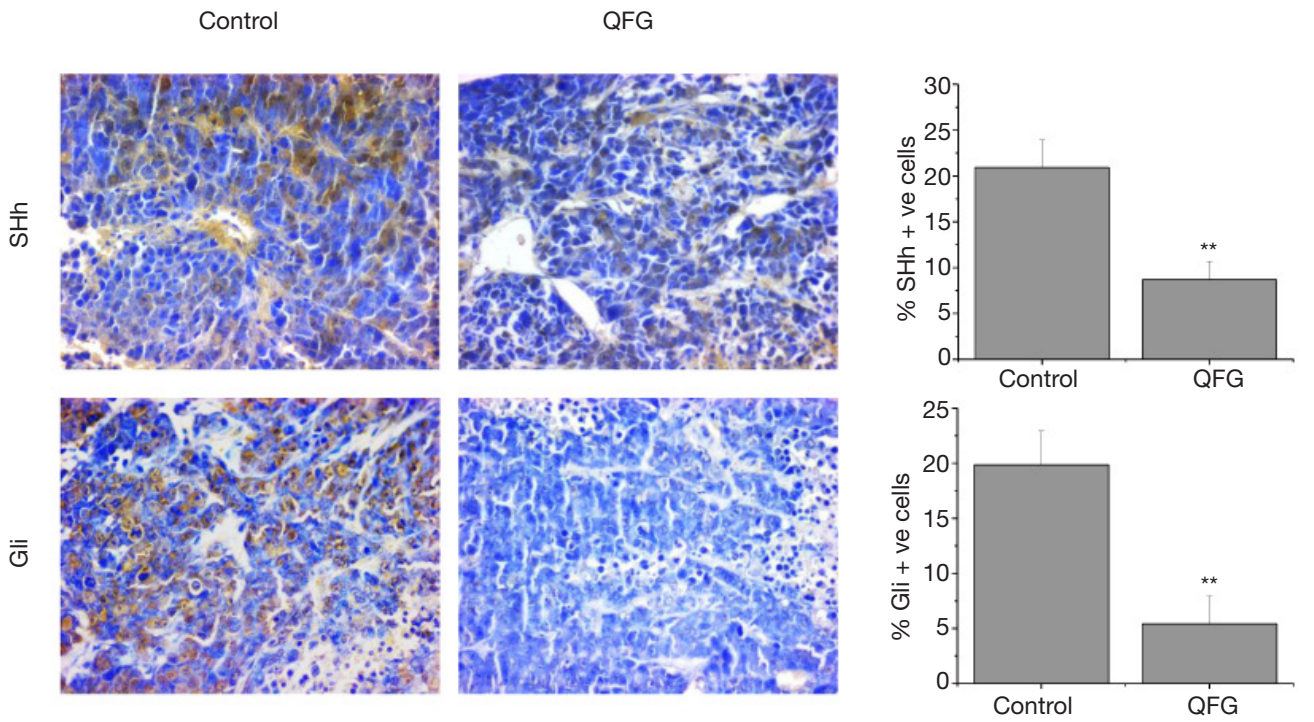

B

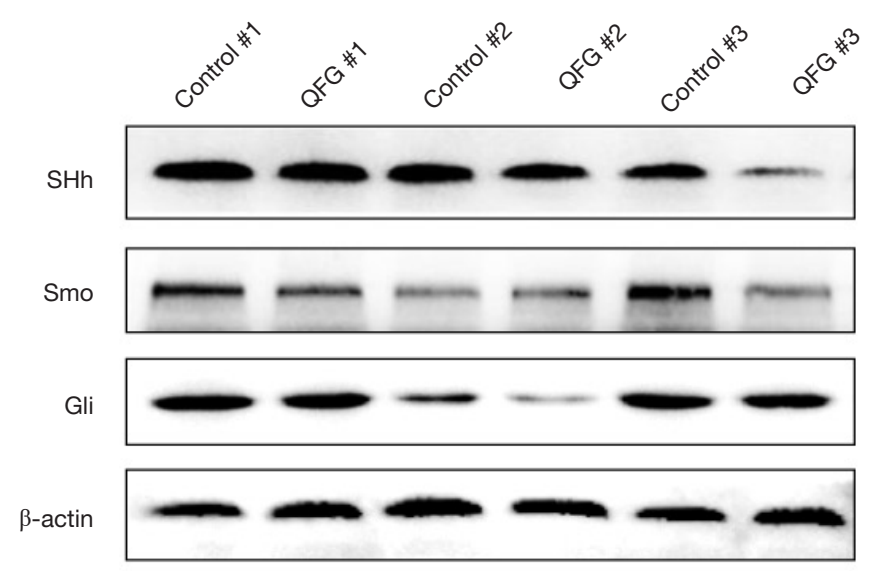

C
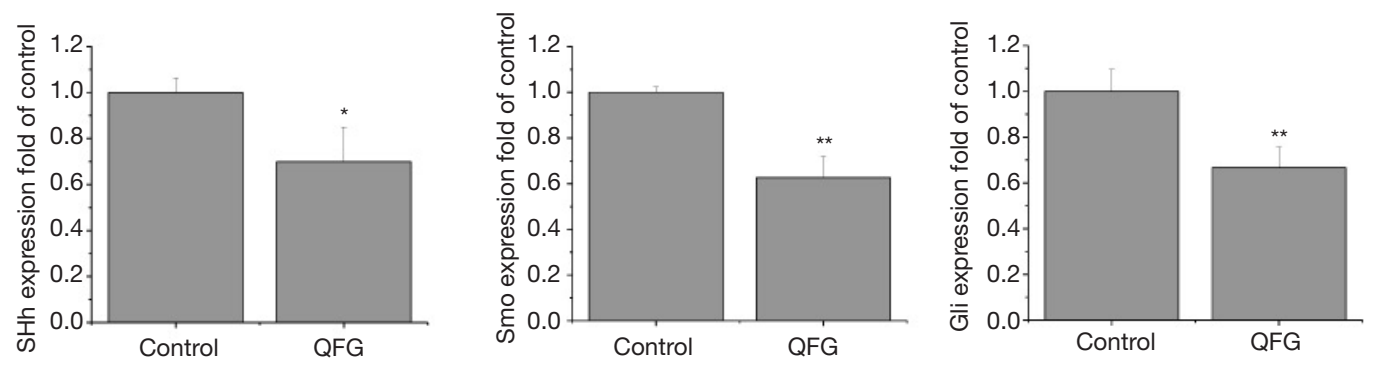

Figure 5 Effect of QFG on the expression of SHh, Smo, Gli in CRC xenograft mice. (A) The expression of SHh and Gli of tumor tissues were detected by IHC assays $(\times 400)$. The percentage of positively stained cells are used as the basis for quantification in IHC assay. Data are expressed as averages with S.D. (error bars), $n=3$ each group. (B) The expression of SHh, Smo and Gli of tumor tissues were detected by Western blot assays. $\beta$-actin was used as an internal control. (C) The band densities of western blots were quantitatively analyzed. Data are expressed as averages with S.D. (error bars), $\mathrm{n}=4$ each group. ${ }^{*} \mathrm{P}<0.05,{ }^{* *} \mathrm{P}<0.01$, QFG treatment group $v$ s. control group. CRC, colorectal cancer; QFG, Qingiie Fuzheng Granules. 
these treatments have side effects and may not improve the quality of life of patients (3). QFG is a traditional Chinese medicine formula for cancer treatment. In clinical research, we found that QFG combined with mFOLFOX4 to treat CRC could raise tumor remission rate, improve immune function, and increase physical fitness and safety (4), but its potential molecular mechanisms in vivo are not yet clear. In the present study, we found that QFG inhibited the growth of tumor volume and weight, which, at the same time, exerted no effect on the body weight in CRC xenograft mice. Thus, it is suggested that QFG effectively inhibited CRC growth in vivo, without no obviously adverse effect on the weight loss of CRC xenograft mice.

It is well known that tumor cell proliferation is one of the key causes of tumor growth. Ki-67 is expressed in proliferating cell nuclei, so is utilized as a proliferation marker (17). In this study, QFG down-regulated the expression of Ki-67 in CRC tumor cells, thus demonstrating that QFG inhibited the proliferation of CRC cells. Cyclin-D1, which is a key protein in the G1 phase of the cell cycle, promotes cell cycle progression from $\mathrm{G} 1$ to $\mathrm{S}$ phase and accelerates cell proliferation by binding to and activating the cyclin-dependent kinase CDK4 (19,25). Survivin is a G2/M phase regulator that promotes the proliferation of transformed cells through mitosis (26). Survivin forms a survivin-CDK4 complex with the cell cycle regulator CDK 4, which releases p21 from the CDK4 complex $(27,28)$. Then, p21 further binds to mitochondrial caspase- 3 and inhibits the activity, thus prevents apoptosis and participates in tumorigenesis (27). Hence, the expressions of survivin, CDK4, Cyclin-D1, and p21 indicate the proliferation state of CRC cells to some degree, whereas p21 possess the contrary effect of CyclinD1-CDK4 on cell proliferation. This research established that QFG up-regulated the expression of p21, but downregulated the expression of Cyclin-D1, CDK4, and survivin. Overall, these results revealed that QFG inhibited the CRC cell proliferation.

Apoptosis is essential for maintaining homeostasis in the body by removing excess and abnormal cells $(29,30)$. Abnormal apoptotic processes can lead to a variety of diseases, such as cancer and autoimmune diseases (29,30). Among the many apoptosis-regulating genes, the caspase and Bcl-2 protein family are most critical. Caspase-3, the most critical apoptosis performer downstream of the caspase cascade, is known as the "death protease" (31). Previous studies have demonstrated that co-expression level of cleaved caspase- 3 (cle-caspase- 3 ) and caspase- 3 might be a prognostic biomarker for cancer patients $(32,33)$. This study demonstrates that QFG increased the expression of cle-caspase-3 in CRC tumor cells and induced apoptosis. In addition to caspase- 3 , the Bcl-2 family is an important regulator of apoptosis (34). The Bcl-2 and Bax gene are a pair of regulatory genes that are known to function in opposition to each other in the process of apoptosis regulation (35). Bcl-2 and Bax affect the sensitivity of tumor cells to various apoptotic stimuli by forming homodimers or heterodimers, and their ratio is the key to ultimately determining cell survival or death (36). When the expression of $\mathrm{Bax}$ is greater than $\mathrm{Bcl}-2$ in the cell, the homodimer is dominant and prone to apoptosis. When expression of Bcl2 is greater than Bax, the heterodimer is increased, and the apoptosis trend is weakened. Studies have found $B a x$ and $\mathrm{Bcl}-2$ gene expression, and $\mathrm{Bax} / \mathrm{Bcl}-2$ ratio may have prognostic and predictive significance in CRC (37). Further, our present study established that QFG up-regulated the expression of Bax and down-regulated the expression of Bcl2 in CRC tumor cells. Overall, these results revealed that QFG induced CRC cell apoptosis.

During the cancer growth and development, angiogenesis plays a vital role. Angiogenesis is the anatomical basis and precondition for the tumor blood supply and the necessary condition for the growth, metastasis, and diffusion of tumors (12). CD31 and vascular endothelial growth factor (VEGF) are the markers of angiogenesis. As a highly expressed marker on the surface of endothelial cells, CD31 can depict the micro vessel density (38). CD31 had been reported to be involved in angiogenesis, for example in the prognosis of CRC cases (39). This study established that QFG suppressed CD31 expression, demonstrating that QFG inhibited CRC tumor angiogenesis. A key factor in the regulation of angiogenesis is VEGF (40). VEGF has different isoforms including VEGF-A, VEGF-B, VEGF-C, and VEGF-D, and has been considered to be the vital factor in promoting angiogenesis and lymphangiogenesis of tumors $(41,42)$. Notably, the biological effects of VEGF are exerted by binding to their receptors VEGFR. VEGFRs that can bind to VEGF are tyrosine protein kinase receptors with three subtypes: VEGFR-1, VEGFR-2 and VEGFR-3 $(41,42)$. Both VEGFR-1 and VEGFR-2 can promote angiogenesis and VEGFR-3 stimulation leads to lymphangiogenesis (43). The signaling effects of VEGF-A ligands binding to VEGFR-2 play an important role in angiogenesis, leading to endothelial cell proliferation, survival, migration and vascular permeability (44). This study demonstrated that QFG down-regulated the expression of 
VEGF-A and VEGFR-2. Briefly, the mechanisms of QFG anti-angiogenesis effects were through the inhibition of the expression of VEGF-A or VEGFR-2.

The pathogenic mechanisms underlying the growth of cancer are heterogeneous and regulated by multiple signaling pathways, including the SHh signaling pathway (13). As one of the crucial intracellular signal transduction pathways, the SHh signaling pathway plays pivotal roles in cell proliferation, apoptosis, and angiogenesis (14). Smo is the key receptor for SHh signaling pathway; only when Smo binds to its corresponding ligands, the signal in $\mathrm{SHh}$ signaling pathway can be transmitted (45). This signal promotes the expression of Gli, which is a downstream target factor in Smo (45). The activated Gli enters the nucleus and controls the transcription of the target gene by binding to the promoter (45). More and more studies have revealed that many effector molecules (such as Cyclin-D, Cyclin-E, survivin, Bcl-2, Bax, caspase-3, caspase-9, c-Myc, VEGF, etc.), involved in tumor cell proliferation, apoptosis and angiogenesis, have been shown to be downstream molecules of the SHh signaling pathway (46-48). It was discovered that MiR-132 regulated the expression of $\mathrm{SHh}$, Cyclin-D1, caspase-3, caspase-9 genes and proteins to promote proliferation and inhibit apoptosis of pancreatic cancer cells through SHh signaling pathway (46). Research by Kim et al. manifested that genipin inhibited proliferation and promotes apoptosis of CRC cells by suppressing $\mathrm{SHh}$ signaling pathway (47). The mechanism may be related to (I) activation of caspase-3, a performer of apoptosis, (II) up-regulation of pro-apoptotic proteins P53, Noxa, Bax, c-caspase-9 and (III) down-regulation of anti-apoptotic proteins surviving and Bcl-2 expression, which both are downstream of SHh signaling pathway (47). Previous studies indicated that Hedyotis diffusa Willd extract inhibited the expression of the VEGF-A as well as VEGFR-2 in a CRC mouse xenograft model to inhibit CRC angiogenesis by suppressing the SHh signaling (48). These findings demonstrated SHh signaling plays an important role in tumor proliferation, apoptosis, and angiogenesis. Therefore, in our study, we speculated that SHh signaling pathway may be the mechanism which allows QFG to inhibit cancer cell proliferation, apoptosis and tumor angiogenesis in a CRC HCT-116 cell mouse xenograft model. We detected the related proteins in the SHh pathway and found that QFG decreased the expression of SHh, Smo and Gli, thereby inhibiting the SHh pathway, regulating the proliferation, apoptosis, and angiogenesis of tumor cells to inhibit the growth of CRC.
The results of this study showed that QFG could inhibit the growth of CRC in CRC xenograft mice. Furthermore, the results of the study have great value in the research of anti-cancer drugs and treatment of clinical patients. However, there was a limitation to the study. CRC Subcutaneous xenograft mouse model has the advantages of screening drugs that inhibit tumor growth, but it is not suitable for screening drugs that inhibit tumor metastasis.

\section{Conclusions}

In conclusion, this study demonstrates that QFG could be valuable for the inhibitory effects against CRC and that it promotes cancer cell apoptosis, inhibits cell proliferation and tumor angiogenesis in CRC by suppressing the SHh pathway, suggesting that QFG may be a potential novel therapeutic drug for CRC.

\section{Acknowledgments}

We thank the financial support from Natural Science Foundation of Fujian Province and Fujian Provincial Health and Family Planning Commission, which enabled this research to be conducted.

Funding: This study was sponsored by Natural Science Foundation of Fujian Province, China (2019J01493), Project Funding for the Training of Young and Middle-aged Backbone Personnel of Fujian Provincial Health and Family Planning Commission (2016-ZQN-67) and Scientific Research Foundation of Traditional Chinese Medicine of Fujian Provincial Health and Family Planning Commission, China (2017FJZYZY203).

\section{Footnote}

Reporting Checklist: The authors have completed the ARRIVE reporting checklist. Available at http://dx.doi. org/10.21037/jgo-20-213

Data Sharing Statement: Available at http://dx.doi. org/10.21037/jgo-20-213

Conflicts of Interest: All authors have completed the ICMJE uniform disclosure form (available at http://dx.doi. org/10.21037/jgo-20-213). The authors have no conflicts of interest to declare.

Ethical Statement: The authors are accountable for all 
aspects of the work in ensuring that questions related to the accuracy or integrity of any part of the work are appropriately investigated and resolved. The animal experimental procedures of this study were performed strictly according to international ethical guide lines and complied with the Guidance Suggestions for the Care and Use of Laboratory Animals issued by the Ministry of Science and Technology of the People's Republic of China. It was approved by Animal Care and Use Committee of Fujian University of Traditional Chinese Medicine (No. 2019009).

Open Access Statement: This is an Open Access article distributed in accordance with the Creative Commons Attribution-NonCommercial-NoDerivs 4.0 International License (CC BY-NC-ND 4.0), which permits the noncommercial replication and distribution of the article with the strict proviso that no changes or edits are made and the original work is properly cited (including links to both the formal publication through the relevant DOI and the license). See: https://creativecommons.org/licenses/by-nc-nd/4.0/.

\section{References}

1. Bray F, Ferlay J, Soerjomataram I, et al. Global cancer statistics 2018: GLOBOCAN estimates of incidence and mortality worldwide for 36 cancers in 185 countries. CA Cancer J Clin 2018;68:394-424.

2. Siegel R, Desantis C, Jemal A. Colorectal cancer statistics, 2014. CA Cancer J Clin 2014;64:104-17.

3. Sun $Y, W u$ X, Zhang Y, et al. Pathological complete response may underestimate distant metastasis in locally advanced rectal cancer following neoadjuvant chemoradiotherapy and radical surgery: incidence, metastatic pattern, and risk factors. Eur J Surg Oncol 2019;45:1225-31.

4. Hua HJ, Lin JM, Ren LP, et al. Effect observation of Qingjie Fuzheng Formula combined with mFOLFOX4 on advanced colorectal cancer treatment. Fujian Journal of Traditional Chinese Medicine 2019;50:20-1.

5. Zhong P, Yang H, Lin S, et al. A Traditional Chinese Medicine Herb Mixture Qingjie Fuzheng Granules Inhibits Hepatocellular Carcinoma Cells Growth by Inducing Apoptosis. J Evid Based Integr Med 2018;23:2515690X18789632.

6. Yang H, Liu JX, Shang HX, et al. Qingjie Fuzheng granules inhibit colorectal cancer cell growth by the PI3K/AKT and ERK pathways. World J Gastrointest Oncol 2019;11:377-92.
7. Zhang L, Jin Y, Peng J, et al. Qingjie Fuzheng Granule attenuates 5-fluorouracil-induced intestinal mucosal damage. Biomed Pharmacother 2019;118:109223.

8. Evan GI, Vousden KH. Proliferation, cell cycle and apoptosis in cancer. Nature 2001;411:342-8.

9. Bold RJ, Termuhlen PM, Mcconkey DJ. Apoptosis, cancer and cancer therapy. Surg Oncol 1997;6:133-42.

10. Reed JC. Dysregulation of Apoptosis in Cancer. J Clin Oncol 1999;17:2941-53.

11. Stoian M, State N, Stoica V, et al. Apoptosis in colorectal cancer. J Med Life 2014;7:160-4.

12. Viallard C, Larrivee B. Tumor angiogenesis and vascular normalization: alternative therapeutic targets. Angiogenesis 2017;20:409-26.

13. Skoda AM, Simovic D, Karin V, et al. A comprehensive review. Bosn J Basic Med Sci 2018;18:8-20.

14. Wicking C, Smyth I, Bale A. The hedgehog signalling pathway in tumorigenesis and development. Oncogene 1999;18:7844-51.

15. Baev DV, Krawczyk J, O'Dwyer M, et al. The BH3mimetic ABT-737 effectively kills acute myeloid leukemia initiating cells. Leuk Res Rep 2014;3:79-82.

16. Zhuang QC, Lin MH, Wei LH, et al. Pien Tze Huang inhibits Colorectal Cancer Cell via Regulating Hedgehog Signaling Pathway in vivo and in vitro. Rehabilitation Medicine 2018;28:21-36.

17. Milde-Langosch K, Karn T, Muller V, et al. Validity of the proliferation markers Ki67, TOP2A, and RacGAP1 in molecular subgroups of breast cancer. Breast Cancer Res Treat 2013;137:57-67.

18. Khan Z, Khan AA, Yadav H, et al. Survivin, a molecular target for therapeutic interventions in squamous cell carcinoma. Cell Mol Biol Lett 2017;22:8.

19. Song HM, Park GH, Park SB, et al. Vitex rotundifolia Fruit Suppresses the Proliferation of Human Colorectal Cancer Cells through Down-regulation of Cyclin-D1 and CDK4 via Proteasomal-Dependent Degradation and Transcriptional Inhibition. Am J Chin Med 2018;46:191-207.

20. Barr AR, Cooper S, Heldt FS, et al. DNA damage during $\mathrm{S}$-phase mediates the proliferation-quiescence decision in the subsequent G1 via p21 expression. Nat Commun 2017;8:14728.

21. Park YG, Choi J, Jung HK, et al. Baicalein inhibits tumor progression by inhibiting tumor cell growth and tumor angiogenesis. Oncol Rep 2017;38:3011-8.

22. Shibuya M. Vascular endothelial growth factor and its receptor system: physiological functions in angiogenesis and pathological roles in various diseases. J Biochem 
2013;153:13-9.

23. Kuipers EJ, Grady WM, Lieberman D, et al. Colorectal cancer. Nat Rev Dis Primers 2015;1:15065.

24. Sarasqueta C, Perales A, Escobar A, et al. Impact of age on the use of adjuvant treatments in patients undergoing surgery for colorectal cancer: patients with stage III colon or stage II/III rectal cancer. BMC Cancer 2019;19:735.

25. Wang H, Chen X, Chen Y, et al. Antitumor activity of novel chimeric peptides derived from Cyclin-D/CDK4 and the protein transduction domain 4. Amino Acids 2013;44:499-510.

26. Ling X, Bernacki RJ, Brattain MG, et al. Induction of survivin expression by taxol (paclitaxel) is an early event, which is independent of taxol-mediated G2/M arrest. J Biol Chem 2004;279:15196-203.

27. Suzuki A, Ito T, Kawano H, et al. Survivin initiates procaspase 3/p21 complex formation as a result of interaction with $\mathrm{Cdk} 4$ to resist Fas-mediated cell death. Oncogene 2000;19:1346-53.

28. Ito T, Shiraki K, Sugimoto K, et al. Survivin promotes cell proliferation in human hepatocellular carcinoma. Hepatology 2000;31:1080-5.

29. Elmore S. Apoptosis: a Review of Programmed Cell Death. Toxicol Pathol 2007;35:495-516.

30. Pistritto G, Trisciuoglio D, Ceci C, et al. Apoptosis as anticancer mechanism: function and dysfunction of its modulators and targeted therapeutic strategies. Aging 2016;8:603-19.

31. Porter AG, Janicke RU. Emerging roles of caspase-3 in apoptosis. Cell Death Differ 1999;6:99-104.

32. Liu PF, Hu YC, Kang BH, et al. Expression levels of cleaved caspase- 3 and caspase- 3 in tumorigenesis and prognosis of oral tongue squamous cell carcinoma. PLoS One 2017;12:e0180620.

33. Perraud A, Akil H, Nouaille M, et al. Implications of cleaved caspase 3 and AIF expression in colorectal cancer based on patient age. Oncol Rep 2012;27:1787-93.

34. Delbridge AR, Grabow S, Strasser A, et al. Thirty years of BCL-2: translating cell death discoveries into novel cancer therapies. Nat Rev Cancer 2016;16:99-109.

35. Brady HJ, Gil-gomez G. Bax. The pro-apoptotic Bcl-2 family member, Bax. Int J Biochem Cell Biol 1998;30:647-50.

36. Saitoh Y, Yaginuma Y, Ishikawa M. Analysis of Bcl-2, Bax and Survivin genes in uterine cancer. Int J Oncol 1999;15:137-41.

37. Khodapasand E, Jafarzadeh N, Farrokhi F, et al. Is Bax/
Bcl-2 ratio considered as a prognostic marker with age and tumor location in colorectal cancer? Iran Biomed J 2015;19:69-75.

38. Basilio-de-Oliveira RP, Pannain VL. Prognostic angiogenic markers (endoglin, VEGF, CD31) and tumor cell proliferation (Ki67) for gastrointestinal stromal tumors. World J Gastroenterol 2015;21:6924-30.

39. Mohamed SY, Mohammed HL, Ibrahim HM, et al. Role of VEGF, CD105, and CD31 in the Prognosis of Colorectal Cancer Cases. J Gastrointest Cancer 2019;50:23-34.

40. Carmeliet P. VEGF as a key mediator of angiogenesis in cancer. Oncology 2005;69 Suppl 3:4-10.

41. Lohela M, Bry M, Tammela T, et al. VEGFs and receptors involved in angiogenesis versus lymphangiogenesis. Curr Opin Cell Biol 2009;21:154-65.

42. Byrne AM, Bouchier-hayes DJ, Harmey JH. Angiogenic and cell survival functions of vascular endothelial growth factor (VEGF). J Cell Mol Med 2005;9:777-94.

43. Su JL, Yen CJ, Chen PS, et al. The role of the VEGF-C/ VEGFR-3 axis in cancer progression. Br J Cancer 2007;96:541-5.

44. Peach CJ, Mignone VW, Arruda MA, et al. Molecular Pharmacology of VEGF-A Isoforms: Binding and Signalling at VEGFR2. Int J Mol Sci 2018;19:1264.

45. Rimkus TK, Carpenter RL, Qasem S, et al. Targeting the Sonic Hedgehog Signaling Pathway: Review of Smoothened and GLI Inhibitors. Cancers (Basel) 2016;8:22.

46. Zhao DW, Hou YS, Sun FB, et al. Effects of miR-132 on proliferation and apoptosis of pancreatic cancer cells via Hedgehog signaling pathway. Eur Rev Med Pharmacol Sci 2019;23:1978-85.

47. Kim BR, Jeong YA, Na YJ, et al. Genipin suppresses colorectal cancer cells by inhibiting the Sonic Hedgehog pathway. Oncotarget 2017;8:101952-64.

48. Lin J, Wei L, Shen A, et al. Hedyotis diffusa Willd extract suppresses Sonic hedgehog signaling leading to the inhibition of colorectal cancer angiogenesis. Int J Oncol 2013;42:651-6.

Cite this article as: Zhu $\mathrm{XQ}$, Yang $\mathrm{H}$, Lin $\mathrm{MH}$, Shang HX, Peng J, Chen WJ, Chen XZ, Lin JM. Qingjie Fuzheng Granules regulates cancer cell proliferation, apoptosis and tumor angiogenesis in colorectal cancer xenograft mice via Sonic Hedgehog pathway. J Gastrointest Oncol 2020;11(6):11231134. doi: 10.21037/jgo-20-213 